\title{
Delay Performance of a Publish Subscribe system deployed over a Memory-Constrained, Delay Tolerant Network
}

\author{
Lorenzo Bracciale*, Donato Battaglino *, Andrea Detti *, Giuseppe Bianchi *, Nicola Blefari-Melazzi *, \\ Andrea Bragagnini ${ }^{\dagger}$, Maura Santina Turolla ${ }^{\dagger}$ \\ *DIE, Universitá di Roma' "Tor Vergata", Rome, Italy \\ \{lorenzo.bracciale,donato.battaglino,andrea.detti,giuseppe.bianchi,blefari\}@ uniroma2.it \\ ${ }^{\dagger}$ Telecom Italia, Turin, Italy \\ \{andrea.bragagnini,maurasantina.turolla@telecomitalia.it\}@telecomitalia.it
}

\begin{abstract}
In this work we assess the delay performance of a Publish Subscribe system built on top of a Delay Tolerant Network (DTN) composed of nodes with limited storage capacities. Many DTN routing protocols replicate the same data over several nodes, in order to deliver data to destination in a faster or in a more reliable way. Of course, increasing the number of replicas has the effect of decreasing the delivery delay perceived by the users, but increases the use of the system memory.

Our goal is to investigate the trade-off between reduction of delay and storage requirements when nodes are memoryconstrained, in a Topic-based, Publish Subscribe system where we have different topics with different popularity.

We provide some insights in this trade-off, which implies some unanticipated issues, and propose simple rules to dimension the number of replicas per topic. To this end, we derive analytical models and we validate them with simulations. ${ }^{1}$.
\end{abstract}

\section{INTRODUCTION}

Publish-Subscribe represents a very popular communication paradigm mainly because of the loose coupling it introduces between actors (publishers / subscribers), which well suits asynchronous and many-to-many communications. One of the most popular publish-subscribe form is the so-called topicbased [1]. In a topic-based publish-subscribe system, users are interested only in a certain set of events, named topics and represented through keywords (e.g. the topic name). Once subscribers have subscribed to a specific topic, they will receive data-samples emitted by publishers on that topic. In other words, topics are logic channels that connect publishers to subscribers.

The publish-subscribe paradigm is a very convenient service model in Delay Tolerant Networks (DTNs). These are networks characterized by intermittent connectivity and/or long propagation delays between the nodes of the network. Typical application scenarios are deep-space, underwater, sensor and tactical networks where connectivity cannot always be guaranteed. Moreover, DTNs well satisfy the requirements of user communities that do not require real time services, e.g. a messaging system in a university campus.

\footnotetext{
${ }^{1}$ This work has been carried out in cooperation with and with funding from Telecom Italia.
}

DTN routing/forwarding schemes employ the so called store-carry-forward paradigm: when connectivity towards a neighbor node is not available, or deemed not convenient by the forwarding scheme employed, a (relay) node temporarily buffer messages. These will be forwarded at a later time, when nodes' movement will make available a link towards the destination. Clearly, the end-to-end delay depends on the mobility model of the nodes (speed, distribution). To improve delay performance and delivery probability, the typical strategy consists in creating multiple copies of a same data-sample over several nodes. Of course, increasing the number of replicas has the effect of decreasing the delivery delay perceived by the users (subscribers) but increases the use of the system memory.

In this paper we present an optimization of a topic-based publish-subscribe system that operates on top of a DTN which uses a multiple copy routing approach. The topics could have different popularity, i.e. different number of subscribers. The DTN is formed by nodes that have a limited memory to store and carry the published data-samples. This case is of interest for DTNs realized with embedded systems such as [2] or, more in general, when the size of data-samples are comparable with the storage space available in the nodes. The optimization goal is to minimize the mean delivery delay perceived by the subscribers.

The presence at the same time of memory constraints and topics with different popularity makes the optimization problem a challenging one. Indeed, as shown later, greedily exploiting all the memory resources available in the network nodes is counter-productive with respect to the system performance. To the best of our knowledge, such issue has not been formerly addressed in the literature. Accordingly, we provide convenient formulae which allow to properly dimension the number of replicas per topic. We further address the case of topics with different popularity and we prove that the optimal number of replicas is proportional to the square root of the topic popularity. We verify the effectiveness of our analytical modeling by means of simulations. To simplify the optimization we preliminarily focus our attention on a 
very simple DTN, employing the spray-and-wait [3] routing scheme, and on random mobility assumptions. However, we believe that our results may be applicable also to other multiple copy routing schemes where the number of replicas per datasample is an input parameter.

\section{PROBLEM DESCRIPTION}

In this section, we introduce the considered scenario and assumptions, we state the delay optimization problem, and we illustrate at a glance the trade-offs that emerge in the optimization.

\section{A. Scenario}

We consider a wireless network composed of $N$ mobile nodes that move within a service area according to a random mobility model. Each mobile node may have publishing and/or subscribing entities for topics; there are $T$ topics and the $i$ th topic has $S_{i}$ subscribing entities. Throughout the paper we refer to a publishing (subscribing) entity with the term publisher (subscriber). Moreover, we refer to the parameter $S_{i}$ as topic popularity.

If a publisher and a subscriber for a same topic are within their radio coverage range, they can directly exchange datasamples. When this is not the case, we assume that datasamples are forwarded throughout the DTN using the "spray and wait" approach. Specifically, the data-samples for the different topics are replicated into the memories of nodes by using the so-called binary spray [3]. Binary spray spreads the replicas of a data-sample for the $i$-th topic as follows: the source generates $R_{i}$ replicas (including the original datasample); any node $A$ with $x>1$ replicas $^{2}$ of the data-sample that meets a node $B$ with no replica forwards $\lfloor n / 2\rfloor$ of its copies to $B$ and keeps the remaining copies.

Unlike [3], we focus on the case of nodes whose memory made available for the DTN forwarding process is bounded. Specifically, we assume that each node may store at most $C_{n}$ data-samples. This sets forth the additional condition that a data-sample is replicated only whenever a neighbor node has at least one memory slot available. The introduction of a memory bound in a topic-based system (where data-samples are persistently generated) implies that a suitable mechanism must be introduced to control the data obsolescence. Viable approaches consist in either defining data-sample lifetimes, or automatic event-based deletion mechanisms, for instance when a node "sees" a newer data-sample for a same topic in a neighbor node [2]. In what follows, for simplicity, we idealize such data obsolescence mechanism by assuming that all the data-samples for a given topic are removed from the memory when a new data-sample is published for that same topic.

Finally, we assume that the time needed to exchange datasample among neighbor nodes is negligible, and that a contact

\footnotetext{
${ }^{2}$ Note that when a node, because of the spray operation, holds $x>1$ replicas for a data-sample, all the $x-1$ copies do not account in the memory occupation of the node, i.e. only one copy of the data-sample is actually stored in the node memory, together with a counter that keeps track of the number of local replicas.
}

among nodes lasts for a sufficient amount of time to exchange all the data-samples that are required by the DTN forwarding rules.

\section{B. Problem Statement and definitions}

We recall that the main characterizing feature of the spray and wait protocol is the number of replicas $R_{i}$ that are disseminated during the spray phase for each data-sample published to the $i$-th topic. In this work, we aim at globally optimizing said number of per topic replicas $\mathbf{R}=\left[R_{1} \cdots R_{T}\right]$, for minimizing the average time $D$ between the publication of a data-sample and its reception by subscribers. We call this delay the mean delivery delay. Obviously, this delay depends on the different topic popularity $S_{i}$. It is readily expressed as the weighted sum of the mean delays $D_{i}$ perceived by subscribers of topic $i$, where the weights are the ratio between the number of subscribers for topic $i$ and the total number of subscribers in the system ${ }^{3}$. In formula:

$$
D=\sum_{i=1}^{T} \frac{S_{i}}{\sum_{j=1}^{T} S_{j}} \cdot D_{i}
$$

It is convenient to express the number of replicas $R_{i}$ in terms of two parameters which we refer to as memory utilization $(\rho)$ and sharing factor $\left(\phi_{i}\right)$. We define the memory utilization $\rho$ as the ratio between the network-wide memory space used to store data-samples $C_{u}=\sum_{i} R_{i}$ and the overall available memory $C_{t o t}=N C_{n}$ :

$$
\rho=\frac{C_{u}}{C_{t o t}}=\frac{\sum_{i=1}^{T} R_{i}}{N C_{n}}
$$

Moreover, for each topic $i$ we define sharing factor $\phi_{i}$ as the fraction of the used memory space $C_{u}$ assigned to topic $i$ for its replicas:

$$
\phi_{i}=\frac{R_{i}}{C_{u}}=\frac{R_{i}}{\sum_{j=1}^{T} R_{j}}
$$

The number of replicas deployed for a given topic $i$ is readily expressed in terms of these two above defined parameters as:

$$
R_{i}=\rho \cdot \phi_{i} \cdot C_{t o t}=\rho \cdot \phi_{i} \cdot N C_{n}
$$

The problem thus consists in determining the optimal set of parameters $\rho$ and $\left\{\phi_{i}\right\}$ so that the delay $D$ given in equation (1) is minimized, under the condition that no node is able to locally store more than $C_{n}$ distinct replicas.

\section{Discussion}

At a first glance, the need to account for the memory utilization $\rho$ may not be evident. Indeed, the per topic average delay $D_{i}$ clearly depends on the number of deployed replicas $R_{i}$, and without memory constraints the delay performance would improve with a larger number of replicas. It could therefore naively appears that the sum of the replicas should

\footnotetext{
${ }^{3}$ We note that every node may be a subscriber for one or more topics, so the number of subscriptions could be greater than the number of nodes. Throughout this paper we consider one subscriber per subscription, so also the number of subscribers could be greater than the number of nodes.
} 
be set to the maximum value allotted by the global memory constrains, i.e., $\rho=1$. However, a closer look reveals that this is not the case. Indeed, the optimal choice derives from tradeoffs that involve both the memory utilization and the sharing of the memory space:

- memory utilization - as we increase the overall number of replicas in the system, the probability that the subscriber meets a node having one of these replicas increases and that tends to reduce the delivery delay. Conversely, as we increase the overall number of replicas in the system, the probability of finding a node with a free memory space decreases. Consequently, the duration of the spray phase is longer, the time required to spread in the system a given number of replicas increases and that tends to increase the delivery delay.

- memory sharing - let we consider a situation where each topic has the same number of replicas and hence all topics fairly share the system memory. As we increase the number of replicas for a given topic $i$, we have to decrement the number of replicas for another topic $j$, so as to maintain constant the memory occupancy. In doing so, the delivery delay of topic $i$ decreases and the delivery delay of topic $j$ increases. This delay unfairness could reduce the overall mean delivery delay $(D)$, when the number of subscribers $S_{i}$ of topic $i$ is greater than $S_{j}$. However, an excessive unfairness could cause so high delay penalty for subscribers of topic $j$ as to vanish the benefits of delay reduction obtained by subscribers of topic $i$.

\section{Problem Resolution}

In the general case of different topic popularity, a delay optimization jointly targeting the parameters $\rho$ and $\left\{\phi_{i}\right\}$ appears numerically cumbersome and this could mine the comprehension of the problems and the derivation of practical guidelines. Therefore, we resorted to a sub-optimal sequential approach which consists in two subsequent steps:

1) memory utilization optimization: first, we derive the memory utilization $\rho$ which minimizes delay in the assumption that all the topics have the same popularity and, hence, $\phi_{i}=1 / T$;

2) sharing factor optimization: second, we derive the sharing factors $\phi_{i}$, by assuming that the available memory is the one derived by the previous memory utilization optimization.

To simplify the analytical computation we consider exponentially distributed inter-meeting time between any pair of nodes. This implies that each node sees another (uniform distributed) random node every $1 / \lambda$ seconds (in mean).

\section{A. Memory Utilization Optimization}

Under the assumption that all the topics have the same popularity and deploy the same number of per topic replica $R=R_{i}$, the subscribers of the different topics experience the same delay that is equal to the mean delivery delay, i.e. $D=D_{i}$. It therefore suffices to study the delivery delay for a

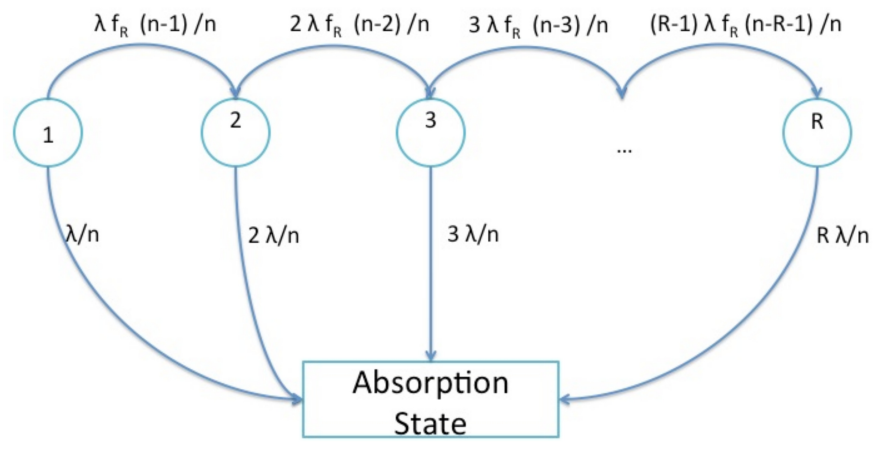

Fig. 1. Markov chain representing the number of replicas in the system for a given data-sample.

single topic, by determining an analytic formula (eq. 8) which expresses the delivery delay as a function of the memory utilization, and numerically find the optimal value of $\rho$ that provides the minimum delay value.

To make the computation of $D$ analytically tractable we resort to the following assumptions:

- we approximate the binary spray mechanisms as an epidemic diffusion of data-sample replicas that stops when the target number of replicas $R_{i}$ is reached.

- we assume only one spray at a time; in other words, during the spray phase of the considered topic, the amount of memory used for data-sample replicas of other topics does not change. This is a good approximation for the case of infrequently emissions of data-samples, whereas it should be considered as a worst case if datasamples production becomes frequent.

We model the evolution of spray and wait using the continuous time Absorption Markov Chain represented in figure 1. The state variable of the Markov chain is the number of deployed replicas of the considered topic. When a data-sample is first produced, the chain starts with state 1 , meaning that only the producer has a copy of the data-sample. Whenever the producer "meets" another node, the chain moves to state 2 , meaning that two nodes in the network carry a data-sample replica. When the chain reaches state $R$, meaning that the datasample is copied over $R$ nodes, the so-approximated spray phase stops.

In addition, a special state called "absorption" state models the reception of the data-sample by a selected subscriber for that topic, hereafter referred to as target subscriber. As such, the average data-sample delivery delay can be simply computed by determining the absorption time in the Markov chain. For ease of notation, we conveniently call $n$ the number of nodes in the network excluding the target subscriber (i.e. $n=N-1)$.

Each state $i \in(1: R-1)$ in the chain has two outgoing transition rates: one towards the state $i+1$, and the other towards the absorption state. These transition rates are derived and justified as follows:

- transition rate $\{i \rightarrow i+1\}$. When the chain is in state 
$i$, a transition to state $i+1$ occurs whenever one of the $i$ replica nodes encounters any of the remaining nodes except the target subscriber. Since the rate of encounters for each node is $\lambda$, the aggregate rate of encounters for the $i$ nodes storing a data-sample replica is $\lambda \cdot i$. Among these encounters, the data-sample is further replicated only when i) the encountered node is not the target subscriber and it does not already hold the replica, and ii) the encountered node has at least one memory slot available to accommodate the replica. Upon an actual encounter, condition (i) occurs with probability $(n-i) / n$, whereas, to model condition (ii), we define $f_{R}$ to be the probability that the encountered node has at least a memory slot available. $f_{R}$ is derived in the subsequent expression (7). We thus conclude that the transition rate $\{i \rightarrow i+1\}$ is expressed by the product $i \lambda f_{R}(n-i) / n$.

- transition rate $\{i \rightarrow$ absorption $\}$. Transition to the absorption state occurs when the target subscriber meets any of the $i$ nodes holding the data-sample. In formula, this is expressed by $i \lambda / n$.

Using this chain, the mean delivery delay $D$ of the considered topic can be calculated as the delay between the state 1 and the absorption state (state 0 ). We define $\Delta_{i}$ and $\delta_{i, j}$ respectively the mean time the system remains in the state $i$ and the average time needed to move from the state $i$ to $j$.

According to the Markov chain theory, the state equations can be written as:

$$
\begin{aligned}
\delta_{1,0}= & \Delta_{1}+\frac{f_{R}(n-1)}{1+f_{R}(n-1)} \delta_{2,0} \\
\delta_{2,0}= & \Delta_{2}+\frac{f_{R}(n-2)}{1+f_{R}(n-2)} \delta_{3,0} \\
\delta_{3,0}= & \Delta_{3}+\frac{f_{R}(n-3)}{1+f_{R}(n-3)} \delta_{4,0} \\
& \cdots \\
\delta_{R, 0}= & \Delta_{R}
\end{aligned}
$$

The mean time $\Delta_{i}$ the system remains in the state $i$ is given by the inverse of the outbound transition rates from each state:

$$
\begin{aligned}
\Delta_{1} & =\frac{n}{\lambda\left[1+f_{R}(n-1)\right]} \\
\Delta_{2} & =\frac{n}{2 \lambda\left[1+f_{R}(n-2)\right]} \\
\Delta_{3} & =\frac{n}{3 \lambda\left[1+f_{R}(n-3)\right]} \\
\Delta_{R} & =\frac{n}{R \lambda}
\end{aligned}
$$

To conclude the analysis, it remains to derive the probability $f_{R}$ used in the chain. This is defined as the probability that an encountered node has a free memory slot to accommodate the replica. We remark that $f_{R}$ does not change with the chain evolution ${ }^{4}$, and can be computed by quantifying the probability

\footnotetext{
${ }^{4}$ We recall that we model the process of data-sample distribution for a given topic, meanwhile assuming that any remaining topic has already "sprayed" its data-sample and thus reached steady state. A joint modeling of all topics would yield an exponential increase in the space state of the chain.
}

that a node has at least one free memory slot before the start of the spray phase, i.e. when there are only $R(T-1)$ replicas in the system generated by the remaining topics. Specifically, note that the replicas generated for the considered topic do not affect the probability $f_{R}$. Indeed, nodes which already hold a replica for the considered topic are not accounted in the term $f_{R}$, but are accounted in the complementary term $(n-i) / n$ within the state transition rate. It follows that $f_{R}$ can be well approximated by:

$$
f_{R}=1-\left(\frac{n}{n+1}\right)^{C_{t o t}-R(T-1)}
$$

This formula is obtained considering that before the start of the spray there are $C_{t o t}-R(T-1)$ memory slots available for the considered topic, distributed on node memories. Considering free memory slots as "tokens" randomly assigned to nodes, the probability that a node has at least a free memory slot is the complementary probability that the node has not been assigned any free space.

Recursively solving equation (7) using the values (6), we obtain the following expression for the mean delivery delay $D=\delta_{1,0}$ as a function of the parameter $R$ :

$$
D=\sum_{k=1}^{R-1} \frac{\prod_{w=1}^{k} \frac{f_{R}(n-w+1)}{1+f_{R}(n-w)}}{\lambda f_{R} k}+n \frac{\prod_{w=1}^{R-1} \frac{f_{R}(n-w)}{1+f_{R}(n-w)}}{\lambda R}
$$

which can be rewritten in terms of the memory utilization $\rho$ (and numerically minimized with respect to such unique variable) by simply substituting:

$$
R=\rho \cdot \frac{C_{t o t}}{T}=\rho \cdot \frac{N \cdot C_{n}}{T}
$$

\section{B. Sharing Factor Optimization}

In this section we derive the optimal sharing factors $\phi_{i}$, under the assumption that the number of memory slots used in the network is fixed to the value $C_{u}=\rho C_{\text {tot }}$, where $\rho$ is the memory utilization value which minimizes expression 8. Although (8) provides a delay expression which accounts for both the spray and the wait phases, to achieve a closedform solution, similar to [3] we approximate the delay by neglecting the spray phase ${ }^{5}$. In this case, as shown in [3] and as a corollary of the previous analysis (i.e. considering $D \approx \delta_{R, 0}=\Delta_{R}$ ) the mean delivery delay for topic $i$ reduces to the very simple expression:

$$
D_{i}=\frac{n}{\lambda R_{i}}=\frac{n}{\lambda C_{u} \phi_{i}}
$$

\footnotetext{
${ }^{5}$ Note that a more precise analysis, though necessarily addressed via numerical means, may be developed by leveraging the results derived in the previous section instead of using the approximation (9). In fact, it is easy to recognize that equation (8) is readily extended to the general case of different per topic delays $D_{i}$ versus the corresponding $\phi_{i}$, as it depends only upon the $\phi_{i}$ value for the considered topic. Such dependence is indeed confined to the exponent in equation (7), where we note that $C_{t o t}-R(T-1)$, in the general case of different popularity, can be rewritten as $C_{t o t}-C_{u}+C_{u} \phi_{i}$.
} 
Consequently, to compute the sharing factors $\phi_{i}$ we need to solve the following constrained optimization:

$$
\begin{gathered}
\min _{\phi} \sum_{i=1}^{T} \frac{n S_{i}}{\lambda C_{u} \phi_{i} \sum_{j} S_{j}} \\
\text { s.t. } \quad \sum_{i} \phi_{i}=1
\end{gathered}
$$

A closed form solution can be accomplished by means of a Lagrange approach. As a result, we obtain the following simple result:

$$
\phi_{i}=\frac{\sqrt{S_{i}}}{\sqrt{S_{1}}+\ldots \sqrt{S_{T}}}
$$

This result states that, for a relatively large number of nodes compared to the number of deployed replicas, i.e. when the spray time is small with respect to the wait time and hence approximation (9) is tight, the number of deployed replicas per topic is proportional to the square root of the topic popularity, namely the number of topic subscribers.

\section{Performance Evaluation}

To validate the effectiveness of the proposed optimization and the modeling results, we developed an ad-hoc event-driven simulator. The simulator has been written from scratch and its code is available at [4]. As reference scenario, we consider $N=100$ nodes that move in an area of $500 \times 500 \mathrm{~m}^{2}$. The radio coverage range is set to $50 \mathrm{~m}$, simulation duration is 500000 seconds. Every node has enough memory to store up to 10 different data-samples. We used movement traces in which the mean inter-meeting time between a specific pair of nodes is 1400 s (i.e. $N / \lambda=1400$, the inter-meeting time between any pair of nodes hence being $1 / \lambda=14 s$ ).

In the simulator data-samples are generated as follows. For each topic, a first data-sample is generated by a randomly chosen publisher node at the start of the simulation (initial random offsets being used to avoid synchronized start). Then, every topic proceeds in parallel by independently generating subsequent data-samples. Each new data-sample is generated by a topic only when all the subscribers have received the previous data-sample. Specifically, after a 300s supplementary time gap at the end of the previous data-sample delivery phase, a new random publisher is selected for the considered topic.

We carried out the three kinds of performance evaluation, aimed at providing insight about the following aspects:

- effectiveness of the overall optimization (memory utilization + sharing factor) with respect to a not-optimized system;

- performance trade-offs related to the memory utilization;

- performance trade-offs related to the sharing factor.

\section{A. Optimization Effectiveness}

We consider nodes moving according to the Random WayPoint (RWP) model [5] with constant speed of $1 \mathrm{~m} / \mathrm{s}$ and a constant pause time of $5 \mathrm{~s}$ to be sure to have a stationary process [6]. We considered 5 scenarios with different number of topics, i.e. $T=30,50,70,90,100$ topics. The topics

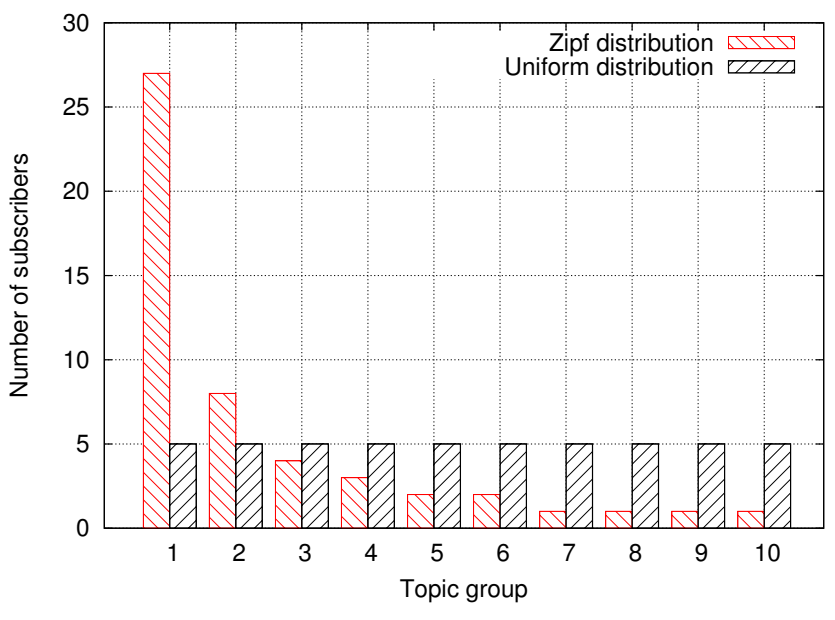

Fig. 2. Number of subscribers per topic
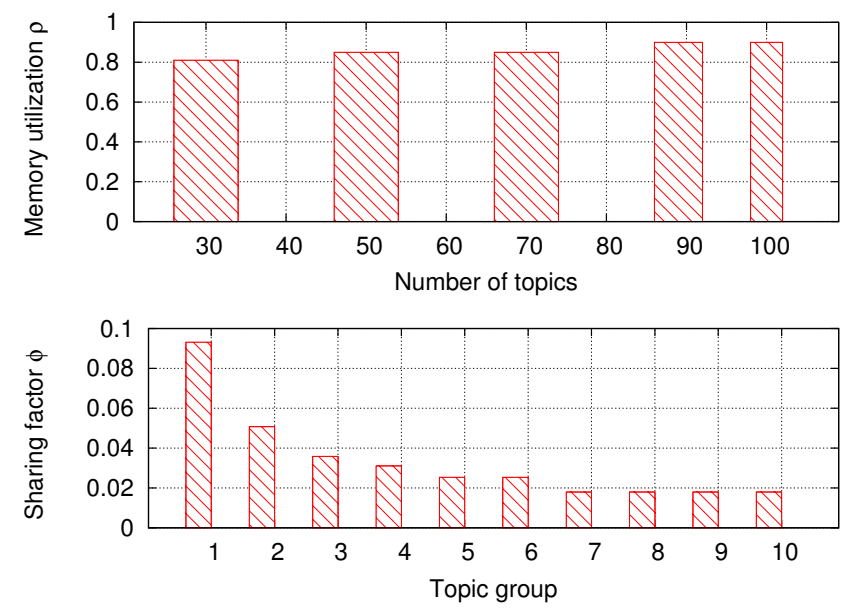

Fig. 3. Memory utilization configuration versus the number of topics and sharing factors in case of 30 topics

have different popularities; we group the topics in 10 different groups of equal size and set the popularity of each group according to a Zipf distribution [7], with parameter $\alpha=0.8$. The topics of the same group have the same popularity. The number of subscribers per topic is reported in figure 2 as a function of the topic group (for instance, a topic of the first group has 27 subscribers).

The subscriptions are uniformly assigned to nodes, so that nodes without any subscriptions are only used for store-carryforward DTN operations.

The upper plot of the figure 3 shows the values of the memory utilization $\rho$ resulting from the optimization versus the different number of topics. We observe that, varying the number of topics, the optimal memory utilization remains in the interval 0.8-0.9; this tell us that it is not convenient to use all the available memory. Setting $\rho$ in this interval can also be considered as a good rule of thumb for these intermediate cases where the number of topics is neither too high nor too low. 
The lower plot of the figure 3 shows the sharing factors $\left\{\phi_{i}\right\}$ of the different topics in case of 30 topics. Those topics are divided in 10 groups ( 3 topics for each group) and popularities of groups are the ones presented in fig. 2. We observe that the sharing factors are proportional to the topic popularities and this proportion depends on the square root of the number of subscriptions as stated in eq. 12 .

In figure 4 we report the comparison of the mean delivery delay for an optimized a non optimized system. In the nonoptimized system, all the available memory is used $(\rho=1)$ and the memory spaces is equally distributed among topics (i.e. $\phi_{i}=1 / T$ ).

We observe that the performance improvement provided by the sub-optimal configuration increases with the number of topics. However, even if it is not reported in the plot, this improvement does not always grow as the number of topic. As an example, if we consider the extreme case of 1000 topics, each topic has the possibility of deploying only 1 replica, thus the optimization returns the same configuration of the non-optimized system (i.e. 1 replica per-topic) and the delay performances are equals.

In general, we argue that the presented optimization is not effective in cases of very little or very large amount of topics (respect to the number of nodes an their capacity), but in the cases of "moderate" number the presented optimization provides a valuable performance improvement. In fact:

- in case of very few amount of topic, both the optimized and non-optimized systems put a large amount of replicas for each topic. This results in good delay performance of the non-optimized system so the improvement carried by the optimization is very limited (see for example the case of 20 topic in figure 6).

- in case of many topics, there are few replication options, e.g., we could only choose between 1 or 2 replicas for a given topic. This means that the optimization process decides on a very limited set of possibilities, and, as a matter of fact, the performance improvement provided by the optimization is limited. For instance, in the worst case of $T=1000, C_{n}=10, N=100$ the only viable solution to diffuse all the data-samples is to use only one replica per topic (i.e., no replication). This configuration is the same of both the optimized and the non-optimized systems.

- in case of a moderate number of topics when the previous conditions do not occurs, the presented optimization provides valuable performance improvements.

\section{B. Memory Utilization Trade-offs}

In this section we analyze the performance trade-offs related to the memory utilization.

We consider scenarios where the topics have the same popularity, then $\phi_{i}=1 / T$. We feed the simulator with two different mobility trace that we call "neg-exp" and "RWP". The former trace is derived considering an exponentially distributed intermeeting times and we use this trace to validate the analytical

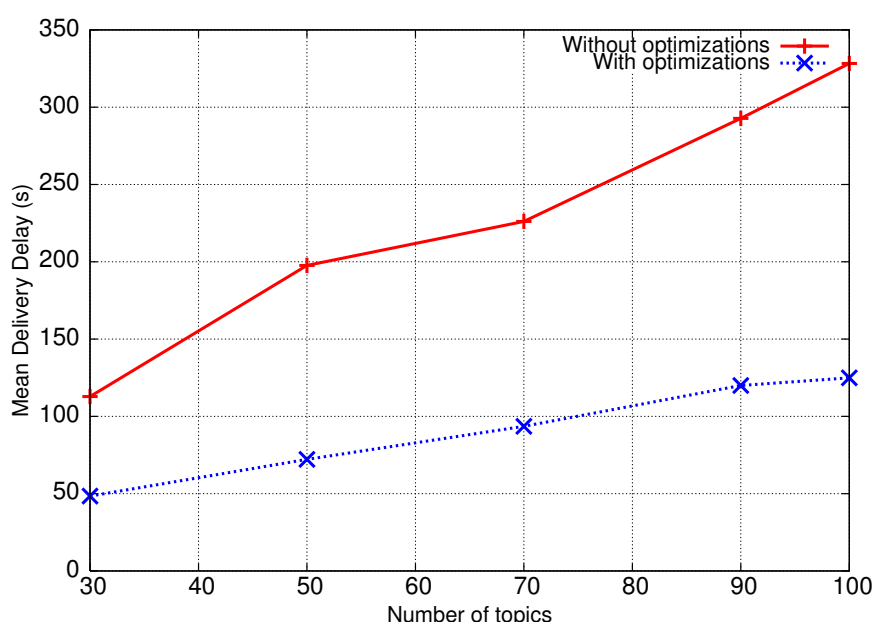

Fig. 4. Mean delivery delay with and without optimal configuration varying the number of the topics

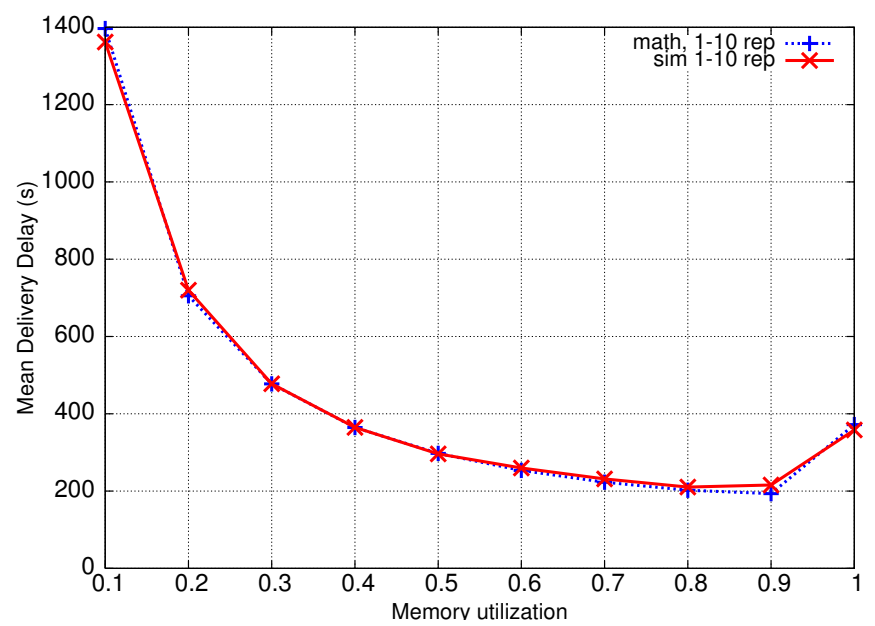

Fig. 5. Mean delivery delay between eq 8 and simulations in the case of 100 topics and varying memory utilization $\rho$

results; the latter trace follows the random waypoint mobility model previously described.

Figure 5 shows a comparison between formula 8 and simulation results in the case of 100 topics and neg-exp movements, varying the memory utilization. The analytical model exhibits a good fitting on the simulation curve. As discussed in section II-C, the graph confirms the presence of an optimum point after which the benefits of introducing more replicas in the system are overwhelmed by a longer spray phase.

Then we changed the mobility trace and consider the RWP movement trace. This allows us to test the usefulness of the optimization on a mobility scenario that is not consistent with the mobility assumptions of the model. Figure 6 shows the mean delivery delay varying the memory utilization and the number of topics. The optimum point varies with the number of topics and the performance worsening due to memory saturation increases with the number of topics, as we can see for instance by comparing the difference between optimal memory utilization and $\rho=1$ for the cases 100 topics and 20 


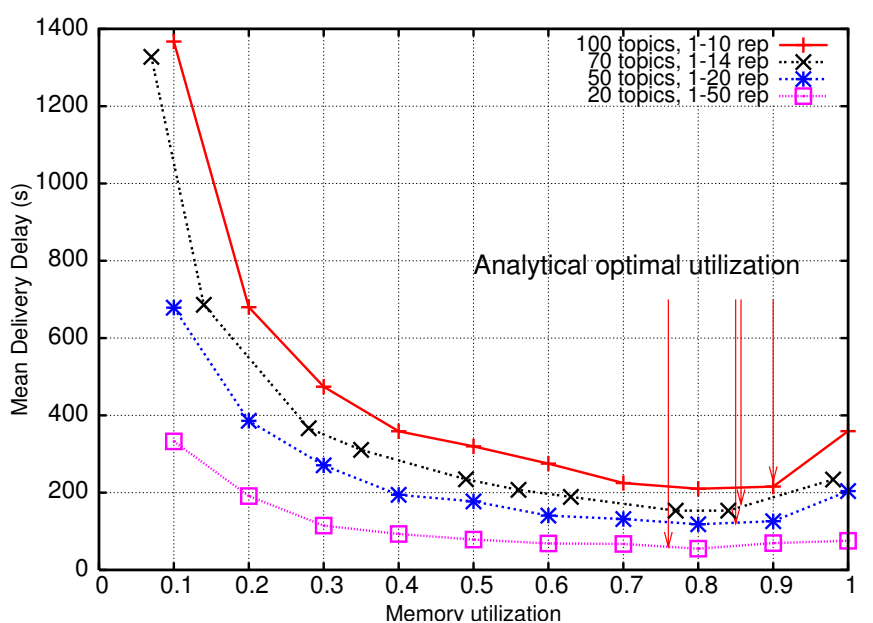

Fig. 6. Mean delivery delay varying the number of topics and memory utilization $\rho$

topics.

The reason is due to the different number of replicas per topic in these two cases, that respectively varies in the ranges 1-10 and 1-50. When we add more replicas in the system, the spray time increases as well. During the spray process, the first replicas are diffused more rapidly, while the latest ones are diffused more slowly. In fact, data-samples can be replicated only on nodes with available memory and that are not already holding a replica for that data-sample. However, when the spray time become significantly high, several replicas are already displaced in the case of 20 topics, while just few replicas are spread for the case 100 topics. For this reason the performance gap between the optimum point and the full memory occupation is smaller in the case of 20 topics and bigger in the case of 100 topics. The graph also shows by arrows the values of memory utilization (i.e. values of the xaxis) returned by the utilization optimization (sec. III-A). As we can see, the optimization succeeds in finding the optimum point in all the presented cases. Graph 7 shows the duration of the spray phase in the same conditions of the previous graph. Here, after a memory utilization of $0.8-0.9$, the spray time curve sharply raises.

\section{Sharing Factor Trade-offs}

In this section we analyze the performance trade-offs related to the sharing factor.

We consider a scenario where we have 100 topics equally divided into two groups, named "popular" and "unpopular" : topics belonging to the popular group are have 50 subscribers, while topics belonging to the unpopular group have 10 subscriber. Nodes move according to a RWP mobility model.

Figure 8 represents the delivery delay of popular and unpopular topics and the overall average delivery delay $(D)$ varying the sharing factor of the popular topics while keeping $\rho=1$. Each point $x$ of the $\mathrm{x}$-axis represents the sharing factor for one popular topic $(1 / 1000 \leq x \leq 9 / 1000)$; in that point each unpopular topic has a sharing factor equal to $(10-x) / 1000$.

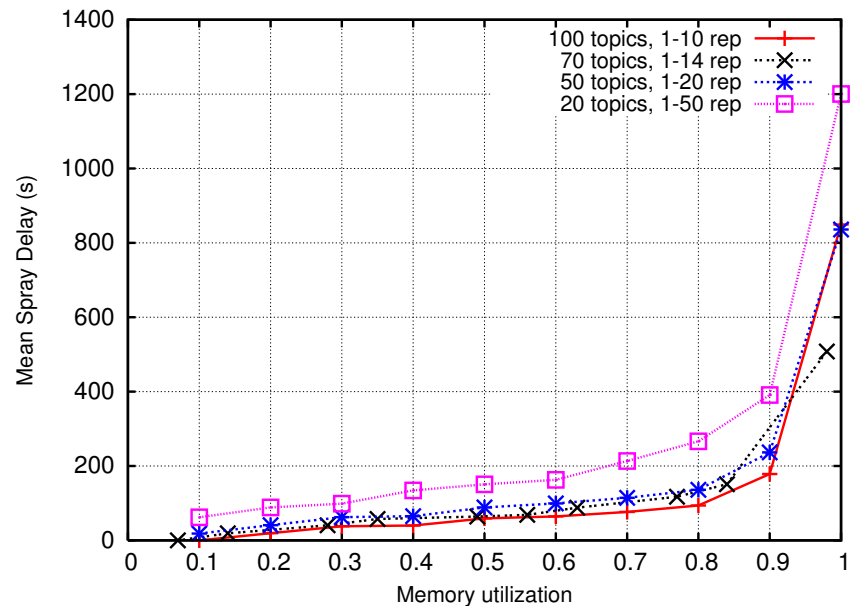

Fig. 7. Mean spray duration time varying the number of topics and the memory utilization

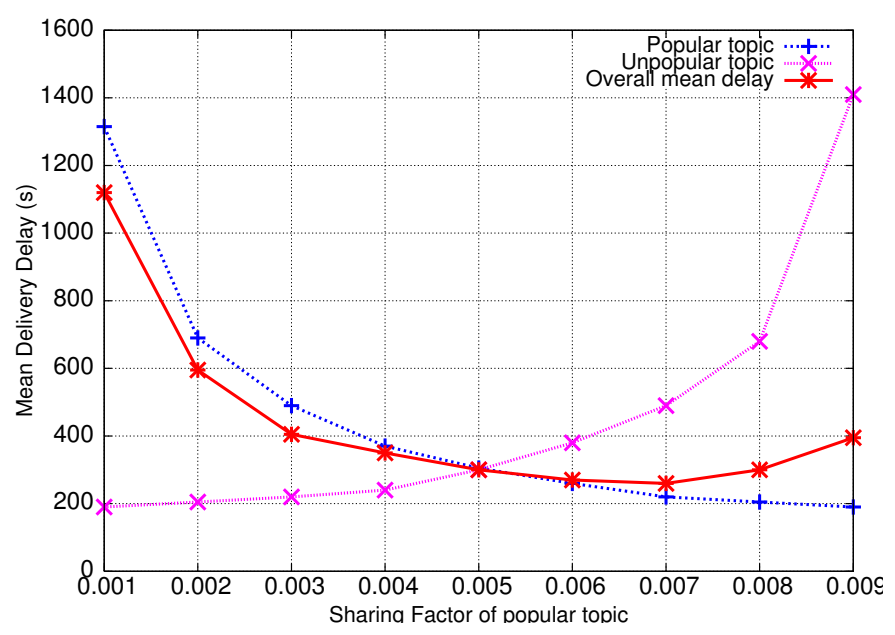

Fig. 8. Mean delivery delay for the case of 100 topics equally divided in two groups, named popular and unpopular

We observe that the minimum of the average delivery delay is achieved with a sharing factor of 7/1000 for popular topic, and $3 / 1000$ to unpopular ones. This result confirms the one given by formula 12 that finds the optimal value in the point 0.00690983 .

\section{RELATED WORK}

Several DTN routing strategies have been proposed so far. If we classify these strategies according to the number or replicas introduced in the network, we have single-copy routing schemes, where only one single copy of a given message is routed and forwarded, and multiple-copy routing schemes, where multiple copies of the same message are routed and forwarded independently.

The single-copy routing scheme proposed in [8] belongs to the first category. In this work, authors propose a routing scheme called "seek and focus" where a single copy of the message is passed by nodes according to an utility function that depends from the latest time a node encounters the 
destination. In the seek phase, if the utility around the node holding the data is "low", the node hands over the data to a neighbor node, with probability $p$ until a node with an high utility is found. When this node is found, the focus phase starts and nodes use utility-based forwarding to deliver the message to the final destination.

With regard to the multiple-copy routing schemes, one possible approach is to distribute a message to all or almost all the nodes in the network. This is the case of [9] where authors propose the so called epidemic routing. This approach consists in distributing messages to node called "carriers" that are within a connected portions of ad-hoc networks. During their movements, carriers will come into contact with another connected portion of the network thus "infecting" additional islands of connected nodes.

The probabilistic routing approach proposed in [10] called PROPHET (Routing Protocol using History of Encounters and Transitivity) aims to overcoming the two main limitations of the epidemic routing approach: the assumption of infinite buffer space and bandwidth in the nodes and the inability of predict user movements.

To overcome such limitations, PROPHET exploits a forwarding strategy based on a probabilistic metric, the "delivery predictability": nodes that are often encountered have a high delivery predictability and delivery predictability reflects the transitive property, according to which if node $a$ frequently encounters node $b$, and node $b$ frequently encounters node $c$, then node $c$ probably is a good node to forward messages destined for node $a$.

There exists also a multiple-copy routing version of the single-copy routing scheme proposed in [8]. This scheme has been presented by the same authors in [3], it is called as "spray and wait" and it is the one we used in this paper. In the same work, the authors propose a second scheme, denoted as "spray and focus", which consists of two different phases as well : i) the spray phase, which is the same as the spray phase in the "spray and wait" scheme, ii) the focus phase, during which each of the $L$ message copy is independently routed according to the single-copy scheme proposed in [8]. In addition, they provide some insight on how to choose the number of replica to spray in the network to achieve a required expected delay (expressed as a multiple of the optimum minimum delay).

For what concern DTN routing used with the publishsubscribe communication paradigm, we cite SocialCast [11]. This work relies on the notion of utility $\mathrm{U}$ of a node $\mathrm{N}$ with respect to interest I, that represents how good a carrier $\mathrm{N}$ is to carry messages matching the interest I. The basic assumption is that hosts which have same interest spend time co-located, so that the SocialCast routing aims at exploiting as carrier for messages hosts which have been co-located often with the interested. Kalman filter forecasting techniques are used to predict the future evolution of the movement based on previous observations on some attributes characterizing social behavior (e.g., connectivity changes, co-location).

\section{COnCLusions}

We tackled the problem of how to optimize the mean delivery delay for a Publish Subscribe system build on top of a Delay Tolerant Network (DTN). We consider scenarios where i) the DTN nodes make available only a limited memory space for routing purposes, and ii) topics have different popularity, i.e. different number of subscribers. We focused on sprayand-wait routing scheme and therefore the only configuration parameter that could be optimized is the number of replicas per topic.

Given that providing an holistic approach results too complex, we split the optimization problem in two parts and we provide answers to these basic questions: i) which is the optimal amount of available memory to use for replication purposes (memory utilization), and ii) which is the right way to share this amount of memory among topics (sharing factor). In doing so, albeit we derived a sub-optimal solution, we have handily and effective formulas. Indeed, with the exclusion of extreme cases of too few or too many topics, the optimization provides a reduction of the mean delivery delay up to $60 \%$, with respect to a non-optimized system

Finally, we argue that our optimization results can be used not only in case of spray-and-wait routing but also in cases of other multiple-copy routing schemes, which have the number of replicas as input parameter. Obviously, in these cases the number of replicas could be just one of the many parameters to optimize.

\section{REFERENCES}

[1] P. T. Eugster, P. A. Felber, R. Guerraoui, A. Kermarrec, The Many Faces of Publish/Subscribe, in ACM Computing Surveys (CSUR), Volume 35, Issue 2, 2003

[2] A. Detti, D. Battaglino, L. Bracciale, F.Lo Piccolo, A. Bragagnini, M.S. Turolla, N. Blefari Melazzi, A topic-based, publish-subscribe architecture for intermittently connected 802.15.4 networks to be presented in Mobile Summit 2011 and available for peer review at http://stud.netgroup.uniroma2.it/ lorenzo/mobilesummit.pdf

[3] T. Spyropoulos , K. Psounis , C. S. Raghavendra, Efficient routing in intermittently connected mobile networks: the multiple-copy case, IEEE/ACM Transactions on Networking (TON), v.16 n.1, p.77-90, February 2008

[4] http://stud.netgroup.uniroma2.it/ lorenzo/campus++/simulator.tar.bz2

[5] T. Camp, J. Boleng, V. Davies, A Survey of Mobility Models for Ad Hoc Network Research, Wiley, Wireless Communications \& Mobile Computing, vol. 2, 2002

[6] J. Yoon, M. Liu, B. Noble, Random waypoint considered harmful, in Proceedings of INFOCOM 2003, San Francisco, April 2003.

[7] Spyros Voulgaris, Etienne Rivire, Anne-Marie Kermarrec, and Maarten van Steen, Sub-2-sub: Self-organizing content-based publish and subscribe for dynamic and large scale collaborative networks, Research Report RR5772, INRIA, Rennes, France, December 2005.

[8] T. Spyropoulos, K. Psounis, and C. S. Raghavendra, Single-copy routing in intermittently connected mobile networks, in Proc. IEEE Conf. Sensor and Ad Hoc Communications and Networks (SECON), 2004, pp. 235244.

[9] A. Vahdat and D. Becker, Epidemic routing for partially connected ad hoc networks, Duke Univ., Durham, NC, Tech. Rep. CS-200006, Apr. 2000.

[10] A. Lindgren, A. Doria, and O. Schelen, Probabilistic routing in intermittently connected networks, SIGMOBILE Mobile Comput. Commun. Rev., vol. 7, no. 3, 2003.

[11] P. Costa, C. Mascolo, M. Musolesi, G. P. Picco, Socially-Aware Routing for Publish-Subscribe in Delay-Tolerant Mobile Ad Hoc Networks, in IEEE Journal of Selected Areas in Communication (JSAC), vol. 26, no. 5, pp. $748-760$, June 2008 